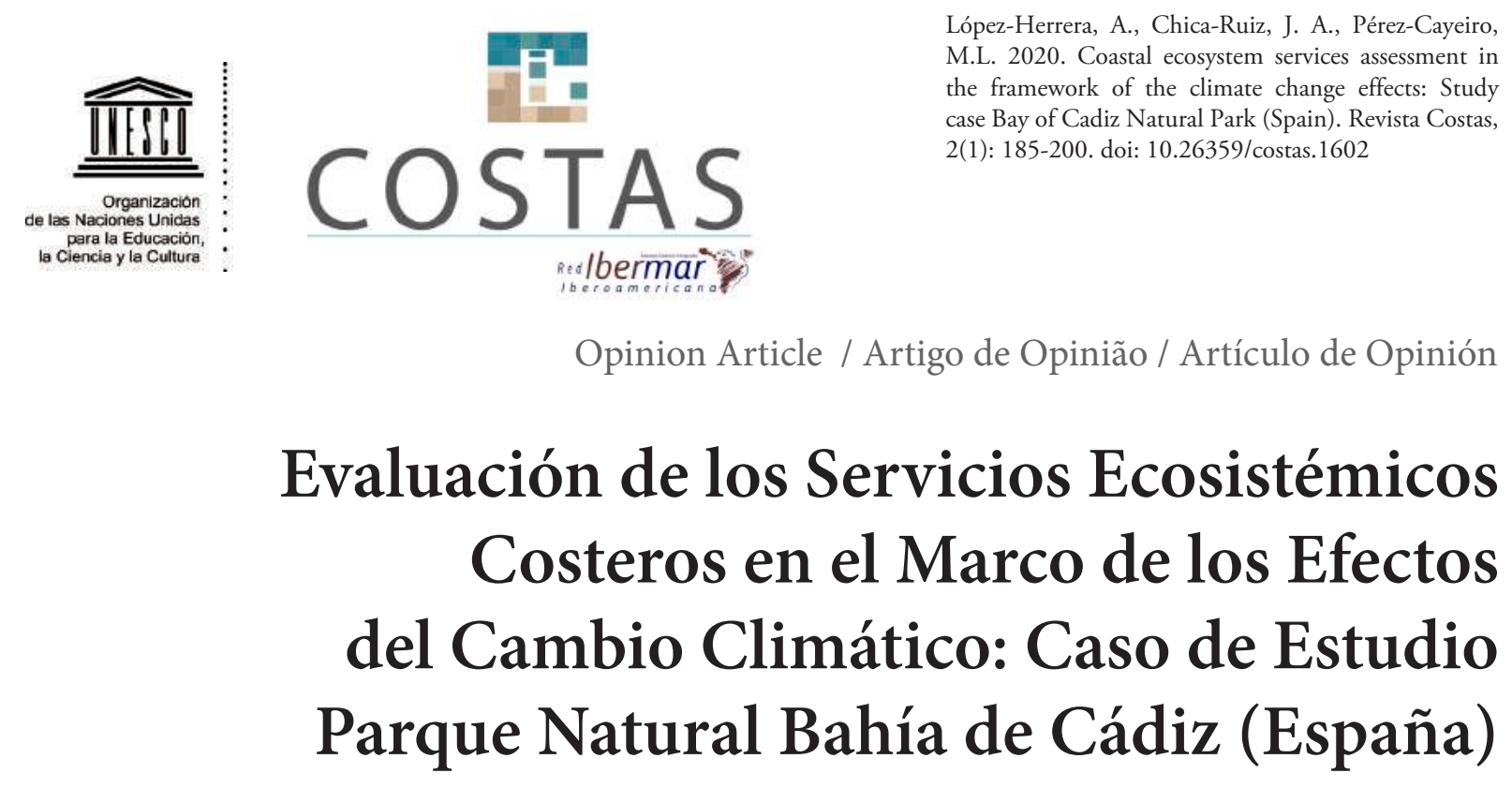

\title{
Coastal Ecosystem Services Assessment in the Framework of the Climate Change Effects: Study Case Bay of Cadiz Natural Park (Spain)
} Alba López-Herrera, J. Adolfo Chica-Ruiz, María Luisa Pérez-Cayeiro e-mail: alba.lopezherre@alum.uca.es

${ }^{1}$ Facultad de Ciencias del Mar y Ambientales. Universidad de Cádiz (España) alba.lopezherre@alum.uca.es adolfo.chica@uca.es isa.perez@uca.es

Submitted: August 2020

Accepted: November 2020

Associate Editor: $\mathrm{xxxxxxxxxxxxxxx}$
Keywords: Protected natural area, ecosystem services, evaluation of ecosystems, human well-being, climate change.

\begin{abstract}
An ecosystem service assessment in the Bay of Cadiz Natural Park has been undergone in relation to the effects of climate change. The ecosystems in the study area were analyzed and 3 different types were indentified: 1) beaches and dunes, 2) marshes and 3) marine environment (including the marine phanerogam meadows), and the ecosystem services (providing, regulating and cultural services) that these ecosystems offer have also been identified. The services state was valued to determine which ones are the most exposed and which the most vulnerable to the climate change effects and its possible future trends through climatic simulators has been made. The results allowed to conclude that most of the ecosystem services showed a negative trend, especially the regulation services which are more associated with the climate change phenomenon.
\end{abstract}




\section{COSTAS}

Revista Costas, Vol. 2 (2), 2020

\section{Resumen}

Se ha realizado una evaluación de los servicios ecosistémicos en el Parque Natural Bahía de Cádiz y su relación con los efectos del cambio climático. Se analizaron los ecosistemas presentes en la zona de estudio, y se identificaron 3 tipos diferentes: 1) playas y dunas, 2) marismas y 3) medio marino (incluyendo las praderas de fanerógamas marinas), e identificado los servicios ecosistémicos (de abastecimiento, de regulación y cultural) que estos ecosistemas ofrecen. Se valoró el estado de los servicios para saber cuáles están más expuestos y son más vulnerables a los efectos generados por el cambio climático y sus posibles tendencias futuras a través de simuladores climáticos. Los resultados permitieron concluir que la mayoría de los servicios ecosistémicos presentaron una tendencia evolutiva negativa, especialmente los de regulación los cuales están más vinculados con el fenómeno de cambio climático.

Palabras claves: Espacio natural protegido, servicios ecosistémicos, evaluación de ecosistemas, bienestar humano, cambio climático.

\section{Introducción}

El concepto de espacio natural protegido (ENP) ha ido cambiando con el tiempo (Tolón \& Lastra, 2008). Inicialmente solo se buscaba proteger y conservar la naturaleza en su estado original, teniendo en cuenta únicamente el valor paisajístico de la zona. No obstante, con el tiempo, se empezó a considerar también la riqueza de especies y hábitats, así como las funciones ecológicas que se realizan en dichos espacios naturales (Gómez, 2011). No hay duda de que la forma de gestión más adecuada es la basada en ecosistemas, según la cual la premisa para establecer medidas de actuación es mantener la integridad de los mismos. Este enfoque es un principio que incide en el reconocimiento de que la naturaleza está integrada y que se debe adoptar una visión holística (UNEP, 2009). Los ecosistemas y la biodiversidad que conforman las áreas protegidas benefician al bienestar humano porque proporcionan una serie de servicios que satisfacen sus necesidades (Costanza et al., 2017).

Desde un punto de vista antropocéntrico se entienden los ecosistemas como un capital natural, lo que indica que estos mantienen su capacidad regenerativa y reproductiva, protegiéndose de las alteraciones (Martín-López et al., 2009) y, sin requerir la acción humana, producen un flujo de servicios a la sociedad (Costanza et al., 2017). De este modo, existe una re- lación entre los ecosistemas y el bienestar humano debido a la generación de estos servicios que satisfacen las necesidades de la población (Martín-López et al., 2009). No obstante, estos servicios no generarían un beneficio social sin la existencia del ser humano (human capital), sus comunidades (social capital) y el entorno donde habitan (built capital) debiendo existir una interrelación de estos capitales con los flujos de servicios ecosistémicos provenientes del capital natural (Costanza et al., 2014).

Según Costanza et al. (2017), los servicios ecosistémicos son aquellos recursos o funciones ecológicas que ayudan al bienestar humano de forma directa o indirecta, o lo que es lo mismo, son aquellos beneficios que obtienen los seres humanos de los ecosistemas.

En el año 2001, las Naciones Unidas dio inicio al programa denominado Evaluación de los Ecosistemas del Milenio (EME) en el que se estudió el estado de los ecosistemas a escala mundial. Se analizaron 24 servicios y se obtuvo que 15 se encontraban degradados o explotados de forma insostenible (Millennium Ecosystem Assessment, 2005).

Posteriormente, en 2007, la Comisión Europea desarrolló un proyecto "la economía de los ecosistemas y la biodiversidad” (TEEB, por sus siglas en inglés). 


\section{COSTAS}

Este informe proporcionó estudios sobre los impactos económicos ocasionados por la pérdida de biodiversidad y la capacidad de mejorarlos (TEEB, 2010).

En 2013 la Agencia Europea de Medio Ambiente (AEMA) publicó la primera versión de la Clasificación Internacional Común de Servicios de los Ecosistemas (CICES, por sus siglas en inglés) que, en 2016, fue modificada. Esta clasificación se creó con el objetivo de tener en cuenta la relación directa existente entre las necesidades del ser humano con los efectos de los ecosistemas (Haines-Young \& Potschin, 2013; 2018). CICES considera los siguientes tipos: de abastecimiento: son todos los productos necesarios para la alimentación, materiales o energía; de regulación o mantenimiento: son aquellos relacionados con la regulación hídrica, del suelo, de la calidad del aire, de flujos, climática o del entorno biótico (Haines-Young \& Potschin, 2013); culturales: son productos no materiales que influyen en el ser humano física, intelectual o espiritualmente, al relacionarse con la naturaleza (Haines-Young \& Potschin, 2018).

No hay duda de que estos servicios pueden verse afectados por las consecuencias ocasionadas por el cambio climático. Este fenómeno se origina a causa del calentamiento global por un aumento de los gases invernaderos en la atmósfera que genera de forma directa un aumento de las temperaturas de la superficie de la tierra (González et al., 2003). El incremento de las temperaturas, junto a otros efectos como cambios en los patrones de precipitación o eventos extre- mos, acaban degradando el medio, causando impactos como la escasez de agua, contaminación en los océanos y modificaciones en la biodiversidad (Díaz, 2012; González et al., 2003; PNACC, 2020).

Hace 18.000 ańos el nivel del mar en la Bahía de Cádiz (Andalucía, España) era menor, con una línea de costa mucho más alejada de la que encontramos en la actualidad. Debido al derretimiento de los polos se generó una subida del nivel del mar dando lugar a inundaciones de llanuras litorales y el retroceso de la línea de costa y la desembocadura del Río Guadalete. El ascenso del nivel del mar favoreció al desarrollo de algunas flechas litorales como la de Valdelagrana o el Barrio Jarana (Puerto Real) fomentando la creación de ambientes estuarinos y marismas (Alonso et al., 2009)

Otro de los efectos producidos por este fenómeno es el aumento de los eventos extremos como los temporales, que afectan fundamentalmente al ecosistema playas y dunas. De igual modo, se han generado daños sobre estructuras marítimas como diques, espigones o paseos marítimos de los municipios que conforman la Bahía de Cádiz y algunos derrumbamientos en los acantilados (La Barrosa, Chiclana de Fra.) (Reyes et al., 1996).

El objetivo del trabajo es realizar una evaluación de los servicios ecosistémicos del Parque Natural Bahía de Cádiz teniendo en cuenta los efectos del cambio climático.

\section{Metodología}

\section{Ambito de estudio}

El estudio ha sido realizado en el Parque Natural Bahía de Cádiz, al suroeste de la Península Ibérica (García-López et al., 2018), entre la desembocadura del río Guadalquivir y el cabo Trafalgar. Su área de influencia socioeconómica incluye cinco térmi- nos municipales: Cádiz, Puerto Real, San Fernando, Chiclana de la Frontera y El Puerto de Santa María. No obstante, estos núcleos urbanos se consideran externos del espacio protegido (PORN, 2004) como se observa en la figura 1 . 


\section{Fis \\ COSTAS}

Revista Costas, Vol. 2 (2), 2020

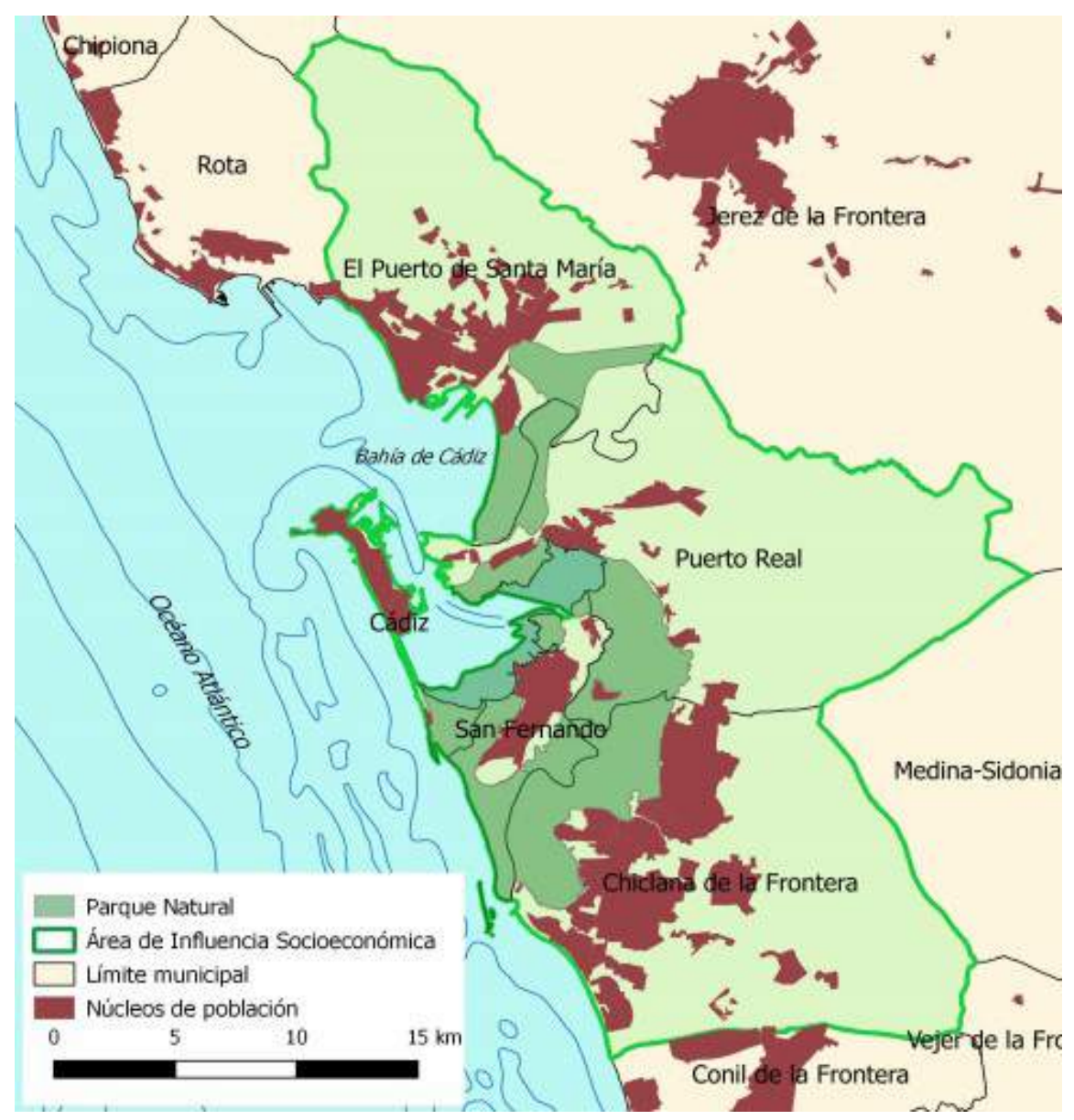

Figura 1. Ámbito territorial de la Bahía de Cádiz. Fuente: CAGPDS, 2019.

Figure 1. Territorial area of the Bay of Cádiz. Source: CAPDS, 2019.

Es una zona con alta importancia ambiental por las distintas unidades existentes, a pesar de estar rodeada de áreas urbanas e industriales, generando un alto interés de conservación desde el punto de vista educativo y científico (García-López et al., 2018).

\section{Metodología}

Se realizó una exhaustiva revisión bibliográfica a partir de diversas fuentes de información. En el trabajo se identifican dos etapas metodológicas:
- Evaluación de los servicios ecosistémicos: se han seleccionado los ecosistemas que mejor definen el área de estudio y se han identificado los servicios ecosistémicos que ofrece cada uno de los ecosistemas. Se localizaron las áreas donde se encuentran y se situaron cartográficamente a través de la plataforma Google Maps y la administración regional ambiental.

- Análisis de los efectos del cambio climático: en esta etapa se realizó un estudio de las zonas afec- 
tadas por los efectos del cambio climático dentro del Parque Natural y los servicios ecosistémicos que serían más vulnerables. Se llevó a cabo un estudio climatológico de la zona a través de $\mathrm{AE}$ MET, para observar la tendencia de las temperaturas, precipitaciones y nivel medio del mar a lo largo de los años. Por otro lado, a través de la plataforma Climate Central, que permite modificar parámetros de tiempo y metros del nivel del mar, se realizaron diferentes simulaciones para observar que ocurriría en un futuro con las áreas estudiadas.

\section{Resultados y discusión}

\section{Análisis de los servicios ecosistémicos}

El Parque Natural Bahía de Cádiz tiene una superficie de 10.522 hectáreas, en la cual se han seleccionado los ecosistemas de mayor interés y se han identificado los servicios que generan: playas y dunas (tabla 1), marismas (tabla 2) y medio marino (tabla 3 ).

El ecosistema playas y dunas, se considera una zona de gran importancia debido a su productividad biológica.

En el entorno del Parque Natural Bahía de Cádiz se desarrollan actividades como la pesca recreativa y de pequeñas embarcaciones. Otra actividad destacada es el marisqueo. Pueden verse afectadas debido a la recolección ilegal, lo que puede crear una sobreexplotación deteriorando el ecosistema (López, 2001).

Los servicios de regulación son esenciales, cumplen la función de control de la erosión y protección frente a eventos extremos y de procesos del ciclo hidrológico (Chica et al., 2011). Sin embargo, son los que más están evolucionando de forma negativa, afectando a la depuración de las aguas o reduciendo la capacidad de amortiguar las perturbaciones (Barragán $\&$ de Andrés, 2020). Los culturales (desarrollo científico y educación ambiental) se están incrementando. Además, el turismo de sol y playa es el sector económico más dinámico.

Por otro lado, el ecosistema marismas son zonas húmedas que se encuentran en continua inundación. Se desarrollan actividades humanas con influencia del mar (de Andrés et al., 2018).
La acuicultura, la pesca extractiva y el marisqueo son actividades con gran influencia económica. Los caños de las marismas son áreas fundamentales para la puesta y cría de especies de interés comercial. Además, se aprovechan antiguas salinas para el sector de la acuicultura. En el ámbito se estiman unas 39 instalaciones activas dedicadas a este sector dentro del Parque Natural. Además de la pesca y el marisqueo, la extracción de sal ha sido otra actividad tradicional de la zona. No obstante, la crisis de dicha actividad dio lugar a desecaciones de marismas destinadas a usos industriales o agrícolas, dejando solo 10 salinas en funcionamiento.

En las playas y dunas, los servicios de regulación han sido alterados por determinadas actividades antropogénicas como la industria o la construcción, fraccionando el ecosistema (desecación de marismas y vertidos contaminantes) (Chica et al., 2011).

También los culturales son de gran relevancia en este ámbito: desarrollo científico y ambiental, valores estéticos y recreativos o patrimonio cultural (casas salineras o los molinos de marea).

Finalmente, el ecosistema medio marino se establece desde la isobata de 50 metros de profundidad hasta aguas profundas (Rodríguez et al., 2011). Este ecosistema está fuera del límite del Parque Natural pero se considera que está dentro de la zona de influencia socioeconómica. Además, son destacables las praderas de fanerógamas dentro de este ecosistema. 
Tabla 1. Servicios suministrados por el ecosistema de playas y dunas.

Table 1. Services provided by the beach and dune ecosystem.

\begin{tabular}{|c|c|c|}
\hline Tipo & Definición & Ejemplos \\
\hline \multicolumn{3}{|c|}{ Servicios de abastecimiento } \\
\hline \multirow[t]{2}{*}{ Alimentación } & $\begin{array}{l}\text { Pesca y Marisqueo para la recolección de } \\
\text { peces, moluscos, crustáceos para alimento. }\end{array}$ & $\begin{array}{l}\text { Langostinos, camarones, almejas, ostras, ostiones, } \\
\text { cañaillas, navajas, bocas, miñocas, gusanas, berberechos, } \\
\text { lubinas, lenguados, lisas, doradas de estero, herreras, } \\
\text { sargos, corvinas, bailas, palometas, jureles, mojarras y } \\
\text { roncaores. }\end{array}$ \\
\hline & $\begin{array}{l}\text { Recolección de algas para uso agrícola } \\
\text { (fertilizantes). }\end{array}$ & $\begin{array}{l}\text { Clorofitas: Ulva Lactuca y Caulerpa prolifera. } \\
\text { Rodófitas: Gigartina acicularis, Lythophylum incrustans y } \\
\text { Gelidium sp. Feofitas: Fucus vesiculosus. }\end{array}$ \\
\hline $\begin{array}{l}\text { Materias primas de } \\
\text { origen geótico }\end{array}$ & Materiales detríticos, geológicos y biológicos. & $\begin{array}{l}\text { Extracción de arenas, barros para baños o conchas para } \\
\text { bisutería. }\end{array}$ \\
\hline $\begin{array}{l}\text { Materiales } \\
\text { medicinales }\end{array}$ & $\begin{array}{l}\text { Agua con sales u otros minerales y especies } \\
\text { vegetales. }\end{array}$ & $\begin{array}{l}\text { Salsola Kali, Cakile maritima, Malcomia littorea, } \\
\text { Eryngium maritimum, Lotus creticus, Helichrysum picardi, } \\
\text { Artemisia crithenifolia y Retama monosperma. }\end{array}$ \\
\hline \multicolumn{3}{|c|}{ Servicios de regulación } \\
\hline $\begin{array}{l}\text { Regulación } \\
\text { morfosedimentaria }\end{array}$ & $\begin{array}{l}\text { Retención y diversificación de los suelos } \\
\text { y control de la erosión. }\end{array}$ & $\begin{array}{l}\text { Cordón de dunas en la playa de Camposoto o playa } \\
\text { del Castillo. }\end{array}$ \\
\hline $\begin{array}{l}\text { Regulación materiales } \\
\text { mar/tierra }\end{array}$ & $\begin{array}{l}\text { Mantenimiento del equilibrio de la } \\
\text { distribución de los materiales } \\
\text { procedentes del mar y tierra. }\end{array}$ & $\begin{array}{l}\text { Playas: Levante, de la Cachucha, del Río San Pedro, de } \\
\text { Camposoto, de Fuentebravía, de Santa Catalina, } \\
\text { de Sancti Petri, de la Barrosa. }\end{array}$ \\
\hline Regulación hídrica & $\begin{array}{l}\text { Diversificación de los flujos } \\
\text { subsuperficiales y subterráneos. }\end{array}$ & Cańo de Sancti Petri. \\
\hline $\begin{array}{l}\text { Regulación } \\
\text { de perturbaciones }\end{array}$ & $\begin{array}{l}\text { Amortiguación a través de áreas donde } \\
\text { se disipa la energía del oleaje. }\end{array}$ & Dunas en la playa de Levante. \\
\hline \multicolumn{3}{|c|}{ Servicios culturales } \\
\hline \multirow{4}{*}{ Paisaje } & \multirow{4}{*}{$\begin{array}{l}\text { Disfrute del entorno paisajístico: ocio, } \\
\text { actividades recreativas y turismo. }\end{array}$} & Mirador del Castillo de Sancti-Petri. \\
\hline & & Centro de visitantes Casa de los Toruńos. \\
\hline & & Centro de visitantes Parque Natural Bahía de Cádiz. \\
\hline & & $\begin{array}{l}\text { Centro de Educación Ambiental y Actividades } \\
\text { en la Naturaleza Coto de la Isleta. }\end{array}$ \\
\hline Estudios científicos & $\begin{array}{l}\text { Investigación de la zona para proyectos } \\
\text { y conocimientos científicos. }\end{array}$ & $\begin{array}{l}\text { Planificación y gestión del litoral; estructura y dinámica } \\
\text { de ecosistemas acuáticos; propiedades físicas de sólidos } \\
\text { amorfos. }\end{array}$ \\
\hline \multirow{3}{*}{ Educación Ambiental } & \multirow{3}{*}{$\begin{array}{l}\text { Aprovechamiento de la zona para desarrollar } \\
\text { actividades de educación ambiental. }\end{array}$} & Centro de los Toruños. \\
\hline & & Centro de visitantes Parque Natural Bahía de Cádiz. \\
\hline & & $\begin{array}{l}\text { Centro de Educación ambiental y Actividades } \\
\text { en la Naturaleza Coto de la isleta. }\end{array}$ \\
\hline Ornamental & Recolección de algas con usos decorativos. & Empresa el Algario. \\
\hline
\end{tabular}




\begin{tabular}{|c|c|c|}
\hline Tipo & Definición & Ejemplos \\
\hline \multicolumn{3}{|c|}{ Servicios de abastecimiento } \\
\hline \multirow[t]{2}{*}{ Alimentación } & $\begin{array}{l}\text { Cría y recolección de especies de } \\
\text { origen marino a través de acuicultura, } \\
\text { pesca extractiva y marisqueo. }\end{array}$ & $\begin{array}{l}\text { Peces: lisas, pez sapo, mojarras y sargos, doradas, lenguados, lubinas, } \\
\text { salemas, corvinas, anguilas, atún rojo. } \\
\text { Moluscos: chocos, pulpos, coquinas de arena o de fango, navajas o } \\
\text { muergos, burgaillos, ostiones, ostras, almejas, berberechos, cañaillas, } \\
\text { y chirlas. } \\
\text { Crustáceos: centollos, cangrejos, gambas, langostinos, camarones, } \\
\text { bocas, cigalas, galeras. } \\
\text { Equinodermos: erizo común. }\end{array}$ \\
\hline & Recolección de algas. & $\begin{array}{l}\text { Algas atlánticas para el sector gastronómico (Salicornia, Aorni verde y } \\
\text { Ulva lactuca). }\end{array}$ \\
\hline $\begin{array}{l}\text { Materias primas } \\
\text { de origen geótico }\end{array}$ & Minerales y áridos. & Sal y arenas. \\
\hline Sistema Energético & $\begin{array}{l}\text { Obtención de energía a través de los } \\
\text { flujos de marea. }\end{array}$ & Molinos de marea (actualmente están en desuso). \\
\hline \multicolumn{3}{|c|}{ Servicios de regulación } \\
\hline $\begin{array}{l}\text { Regulación } \\
\text { morfosedimentaria }\end{array}$ & $\begin{array}{l}\text { Distribución de los sedimentos y } \\
\text { balance sedimentario. Regulación de la } \\
\text { dinámica costera. Controlar la erosión. }\end{array}$ & $\begin{array}{l}\text { Caños: Sancti Petri, Talanquera, del Bote, El Trocadero, del Horcajo, } \\
\text { de la Calavera, del Río San Pedro, entre otros. } \\
\text { Marismas: los Toruños, Sancti Petri, El Trocadero, de Carboneros, de } \\
\text { Cetina, en la playa de levante y del Río San Pedro. }\end{array}$ \\
\hline Regulación hídrica & $\begin{array}{l}\text { Interconexión entre distintas masas de } \\
\text { agua y equilibrios salinos. }\end{array}$ & Río de San Pedro \\
\hline $\begin{array}{l}\text { Regulación de las } \\
\text { perturbaciones }\end{array}$ & $\begin{array}{l}\text { Defensa contra agentes y desastres } \\
\text { naturales. }\end{array}$ & \multirow{2}{*}{$\begin{array}{l}\text { Marismas: los Toruńos, Sancti Petri, El Trocadero, de Carboneros, de } \\
\text { Cetina, en la playa de levante y del Río San Pedro. }\end{array}$} \\
\hline Regulación biológica & $\begin{array}{l}\text { Interacción de las distintas especies en } \\
\text { la cadena trófica. }\end{array}$ & \\
\hline \multicolumn{3}{|c|}{ Servicios culturales } \\
\hline Paisaje & Actividades recreativas, turismo, ocio. & $\begin{array}{l}\text { Observatorios de aves: Carboneros (Chiclana de la Fra.), Eucaliptos, } \\
\text { Río Arillo y Tres Amigos (San Fernando). } \\
\text { Miradores: salina La Esperanza y Los Toruños. } \\
\text { Jardín botánico. } \\
\text { Coto de la Isleta. }\end{array}$ \\
\hline Desarrollo científico & $\begin{array}{l}\text { Elaboración de proyectos y estudios } \\
\text { científicos. }\end{array}$ & $\begin{array}{l}\text { Planificación y gestión del litoral; biología marina y pesquera; } \\
\text { conservación de humedales costeros; de aislamiento, determinación } \\
\text { estructural y síntesis de productos naturales; química biológica. }\end{array}$ \\
\hline $\begin{array}{l}\text { Educación } \\
\text { Ambiental }\end{array}$ & $\begin{array}{l}\text { Aprovechamiento de la zona para } \\
\text { desarrollar actividades de educación } \\
\text { ambiental. }\end{array}$ & $\begin{array}{l}\text { Campañas de protección de casas salineras y molinos de marea (San } \\
\text { Fernando). } \\
\text { Campaña de educación ambiental "conoce nuestros Parques” y } \\
\text { campańas de voluntariado ambiental. }\end{array}$ \\
\hline $\begin{array}{l}\text { Estudio ecológico } \\
\text { local }\end{array}$ & Población en las vías pecuarias. & $\begin{array}{l}\text { El Cordel de El Puerto de Santa María a San Fernando. } \\
\text { La Vereda de Cádiz, desde la Punta del Boquerón a La Ardila en San } \\
\text { Fernando. }\end{array}$ \\
\hline Desarrollo cultural & Disfrute de patrimonios culturales. & Molinos de marea, casas salineras y baterías. \\
\hline
\end{tabular}


Tabla 3. Servicios suministrados por el ecosistema de zona marina

Table 3. Services provided by the marine environment ecosystem.

\begin{tabular}{|c|c|c|}
\hline Tipo & Definición & Ejemplos \\
\hline \multicolumn{3}{|c|}{ Servicios de abastecimiento } \\
\hline \multirow[b]{2}{*}{ Alimentación } & $\begin{array}{l}\text { Actividades como la pesca profesional } \\
\text { o deportiva, marisqueo y recolección } \\
\text { de especies para acuicultura. }\end{array}$ & $\begin{array}{l}\text { Doradas, lubinas, lenguados, langostinos, ostiones, anguilas, alme- } \\
\text { jas, chirlas, navajas, coquinas de fango, galeras, camarones } \\
\text { y cangrejos. }\end{array}$ \\
\hline & $\begin{array}{l}\text { Fanerógamas marinas como zona } \\
\text { de alimento y alevinaje para especies }\end{array}$ & $\begin{array}{l}\text { Clorofitas: Ulva Lactuca y Caulerpa prolifera. } \\
\text { Rodófitas: Gigartina acicularis, Lythophylum incrustans y Gelidium } \\
\text { sp. Feofitas: Fucus Vesiculosus. } \\
\text { Diatomeas y fanerógamas del género Zostera (Cymodocea nodosa). } \\
\text { Praderas de Zostera nolitii y Caulerpa prolifera. }\end{array}$ \\
\hline $\begin{array}{l}\text { Materias primas de } \\
\text { origen geótico }\end{array}$ & $\begin{array}{l}\text { Zona de sedimentación y acumulación } \\
\text { de materiales detríticos geológicos. }\end{array}$ & Arena para regenerar las playas. \\
\hline Transporte & $\begin{array}{l}\text { Uso del medio marino para el transporte } \\
\text { marítimo. }\end{array}$ & Puerto Bahía de Cádiz. \\
\hline \multicolumn{3}{|c|}{ Servicios de regulación } \\
\hline Morfosedimentaria & Control erosión y sedimentación. & $\begin{array}{l}\text { Las praderas de fanerógamas disminuyen la velocidad de las } \\
\text { corrientes: Cymodocea nodosa, Zostera noltei y Zostera marina. }\end{array}$ \\
\hline \multirow[t]{2}{*}{ Hídrica } & Regulación del agua. & $\begin{array}{l}\text { Conexión de la bahía con mar abierto con intercambio de masas } \\
\text { de agua del golfo de Cádiz (externa) y flujo y reflujo de corrientes } \\
\text { de aguas por el Estrecho de Puntales y el Caño de Sancti Petri } \\
\text { (interna). }\end{array}$ \\
\hline & Tratamiento de residuos en el agua. & $\begin{array}{l}\text { Las praderas de fanerógamas captan gran parte de los nutrientes } \\
\text { que se encuentran en el agua: Cymodocea nodosa, Zostera noltei } \\
\text { y Zostera marina. }\end{array}$ \\
\hline Biológica & $\begin{array}{l}\text { Regulación del medio marino para } \\
\text { aportar nutrientes al medio, regular } \\
\text { el control biológico, etc. } \\
\text { Regulación de interacciones entre niveles } \\
\text { tróficos. }\end{array}$ & $\begin{array}{l}\text { Incremento de la biodiversidad por parte de las praderas } \\
\text { de fanerógamas: Cymodocea nodosa, Zostera noltei y Zostera marina } \\
\text { al suministrar refugio y zonas de producción a organismos. }\end{array}$ \\
\hline Climática & $\begin{array}{l}\text { Captura del } \mathrm{CO}_{2} \text { y producción } \\
\text { de oxígeno. }\end{array}$ & $\begin{array}{l}\text { Las praderas de fanerógamas (Cymodocea nodosa, Zostera noltei y } \\
\text { Zostera marina) actúan como sumidero y reservorio de carbono. } \\
\text { Además, liberan oxígeno al medio. }\end{array}$ \\
\hline \multicolumn{3}{|c|}{ Servicios culturales } \\
\hline Paisaje & $\begin{array}{l}\text { Usos recreativos, ocio, actividades } \\
\text { náuticas, pesca deportiva, turismo. } \\
\text { Apreciación del paisaje de forma estética, } \\
\text { hábitat de aves costeras. }\end{array}$ & $\begin{array}{l}\text { Paseos en barco y cruceros por el Pantalán de la Magdalena } \\
\text { (San Fernando), Bahía de Cádiz, Isla Verde, Isla del Trocadero, } \\
\text { Isla Vicario, Río San Pedro, Caño de Sancti Petri. } \\
\text { Vela por toda la Bahía de Cádiz y buceo en las playas } \\
\text { de Fuentebravía y Sancti Petri. } \\
\text { Piragüismo por el descenso del Guadalete. } \\
\text { Pesca y concursos de pesca deportiva. }\end{array}$ \\
\hline $\begin{array}{l}\text { Desarrollo científ- } \\
\text { ico }\end{array}$ & $\begin{array}{l}\text { Uso del ambiente para investigación. } \\
\text { Estudio de las propiedades de las } \\
\text { fanerógamas. }\end{array}$ & $\begin{array}{l}\text { Planificación y gestión del litoral; estructura y dinámica de } \\
\text { ecosistemas acuáticos; biología marina y pesquera: de oceanografía } \\
\text { y contaminación del litoral; de química biológica. }\end{array}$ \\
\hline $\begin{array}{l}\text { Educación } \\
\text { Ambiental }\end{array}$ & $\begin{array}{l}\text { Conocimiento de estas áreas y su } \\
\text { importancia para una mejor gestión. }\end{array}$ & $\begin{array}{l}\text { Voluntariados y prácticas escolares de educación ambiental desde } \\
\text { FAMAR (Red de Voluntarios del Parque Natural Bahía de Cádiz) }\end{array}$ \\
\hline
\end{tabular}


En la Bahía de Cádiz se pueden localizar 3 de las 4 especies que existen en Europa (Cymodocea nodosa, Zostera noltei y Zostera marina) (Hernández et al., 2010).

Como se puede observar, los ecosistemas costeros como playas, dunas y marismas son aquellos de los que se obtiene un mayor número de servicios para la población tanto de regulación como culturales, destacando los relacionados con el sector turístico. No obstante, también son numerosos los servicios de abastecimiento que proporcionan alimentos y materiales necesarios para el bienestar humano.

Según Barragán y de Andrés (2020) en el último estudio realizado acerca de la evolución de los ser- vicios ecosistémicos de la zona, se observa como los de abastecimiento, con prácticas tradicionales como la pesca o el marisqueo, están en detrimento por el avance de técnicas industriales como en la acuicultura. No obstante, los de regulación tienen una mayor tendencia negativa, ya que están relacionados directamente con el clima. Por último, los culturales aumentan como por ejemplo los numerosos estudios científicos que se realizan en la zona o desarrollo de actividades relacionadas con la educación ambiental y el patrimonio cultural.

En la figura 2 se muestra una visión general de los servicios ecosistémicos ofrecidos por el Parque Natural Bahía de Cádiz.

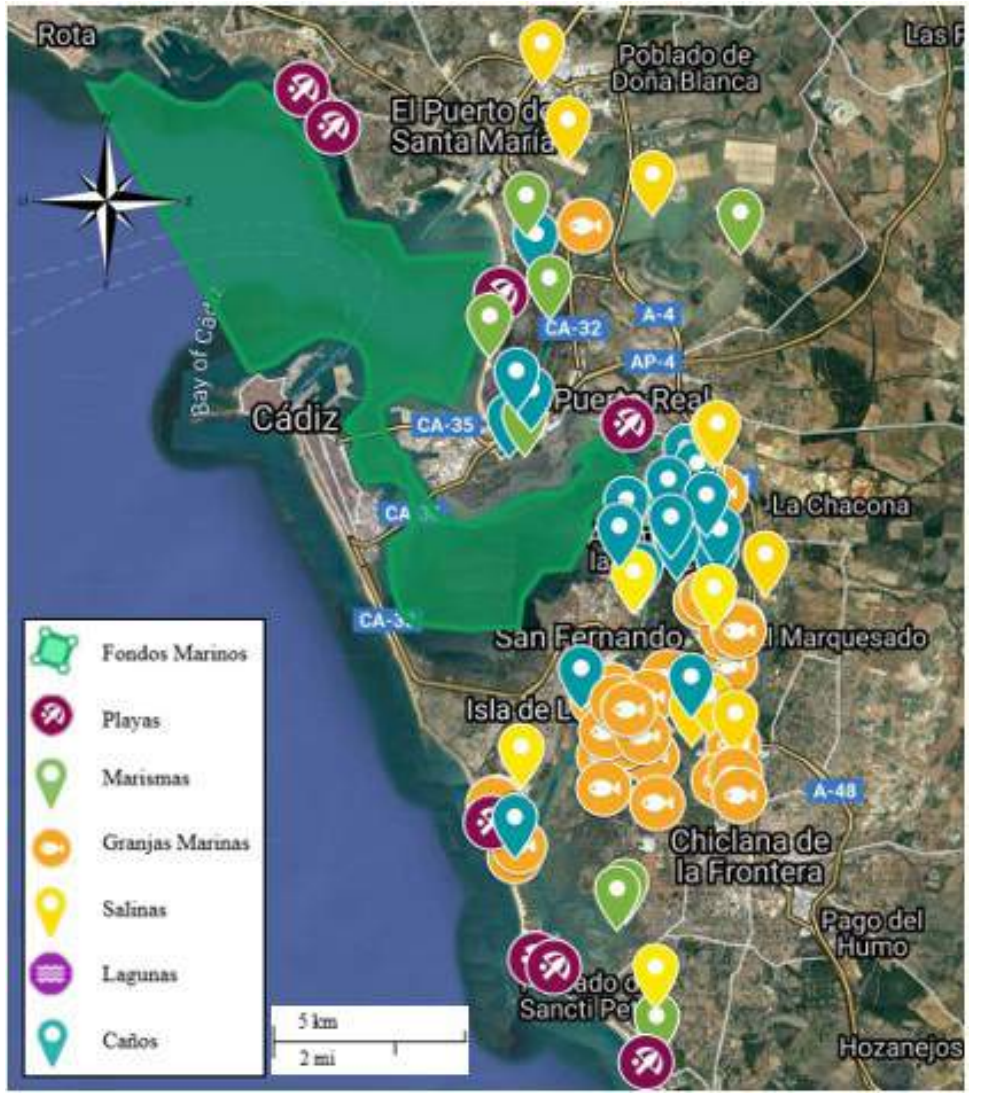

Figura 2. Mapa satélite de los servicios ecosistémicos ofrecidos por el Parque Natural Bahía de Cádiz. Fuente: elaboración propia a través de Google My Maps.

Figure 2. Satellite maps of the ecosystem services provided by the Bay of Cádiz Natural Park. Source: Own elaboration by Google My Maps. 


\section{里 \\ COSTAS}

\section{Efectos del cambio climático}

La Bahía de Cádiz presenta, actualmente, valores climáticos diferentes a los que existían a finales de los 60 y principios de los 70 , donde comenzó a surgir una conciencia real sobre esta amenaza. Si se observan los datos proporcionados por AEMET, en el periodo 1981-2010, la temperatura media anual se encontraba en $18,6^{\circ} \mathrm{C}$. En el periodo 2013-2019, la temperatura se incrementa a $19,1^{\circ} \mathrm{C}$. Por el contrario, en el periodo 1981-2010, se registra una precipitación anual media de $523 \mathrm{~mm}$, sin embargo, entre los años 2013-2019, ha habido un descenso cuya precipitación media fue registrada en $477 \mathrm{~mm}$.

El aumento de las temperaturas puede generar una mayor evaporación de los ambientes acuáticos, lo que conduce a una reducción de algunos hábitats o incluso modificaciones en la calidad del agua (Anderson et al., 2012). Esto, junto al descenso de las precipitaciones, puede provocar un déficit de los recursos hídricos (Ceballos et al., 2007), un aumento de la sa- linidad de estas aguas o un aumento de las emisiones de carbono a los ecosistemas (Anderson et al., 2012) generando pérdidas de hábitat y biodiversidad o afectando a la agricultura y turismo de la zona (Chica et al., 2011). Por otro lado, como consecuencia de estas variaciones climáticas, se producen cambios en los polos dando lugar al derretimiento de los glaciares generando un aumento del nivel del mar. Este es uno de los impactos que más afecta a la zona costera ya que quedan expuestas a inundaciones (Caballero et al., 2007).

En el caso de la Bahía de Cádiz, a lo largo de los años ha ido aumentando el nivel del mar, con algunas variaciones durante el periodo 1961-2018 (figura 3), con valores pico en los últimos años. La tendencia general es ascendente y realmente apreciable la diferencia de los msnm presentados en los 60 (en torno a los $6770 \mathrm{msnm}$ ) a lo que podemos observar hoy día (superior a $6900 \mathrm{msnm}$ ).

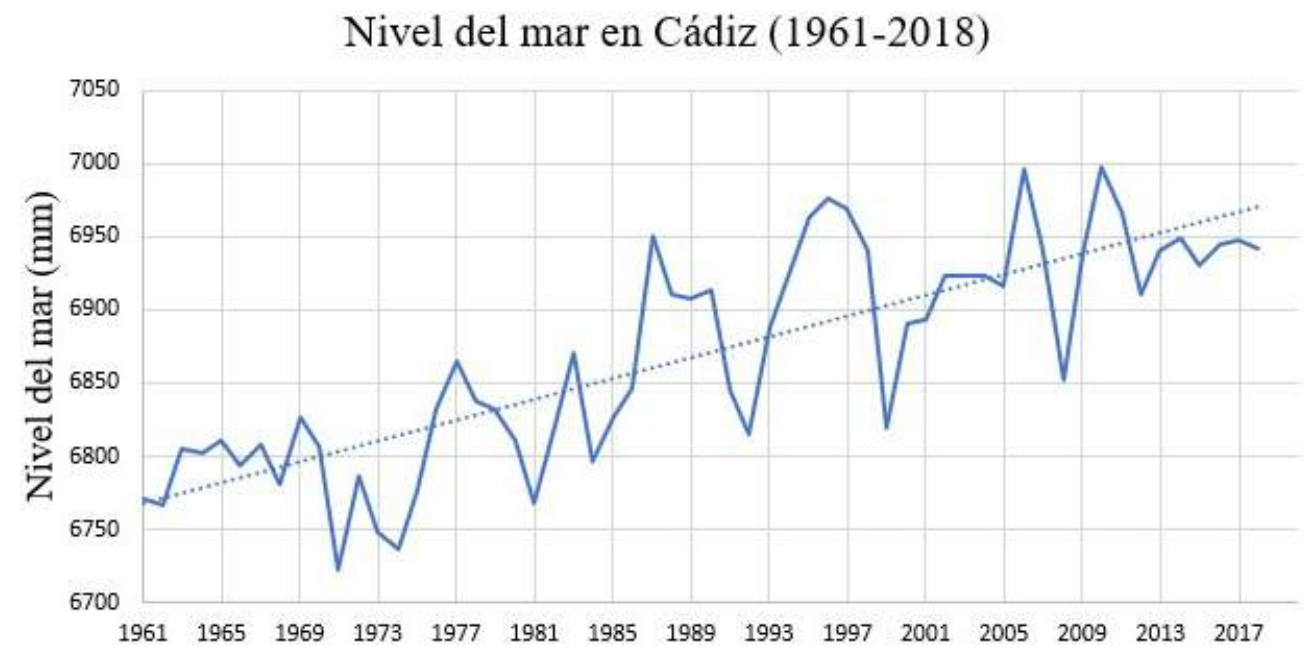

Figura 3. Evolución del nivel del mar (msnm) en Cádiz a lo largo de los ańos desde 1961 a 2018. Fuente: elaboración propia a través de los datos obtenidos en Permanent Service for Mean Sea Level (Mareógrafo Cádiz III).

Figure 3. Sea level development (msnm) in Cadiz over the years from 1961 to 2018.

Source: Own elaboration by data collected in Permanent Service for Mean Sea Level (Tide Gauge of Cadiz III). 
En este estudio se han realizado distintas simulaciones modificando algunos parámetros: año y metros por debajo del nivel del mar. Los mapas simulados muestran las zonas que están expuestas a inundación. En primer lugar, se observa cómo cada vez hay mayor número de zonas influenciadas a medida que variamos los metros de agua por debajo de los cuales el terreno quedaría inundado. Si se analiza la figura 4, ya empezarían a verse afectados algunos servicios ecosistémicos con aumentos de 0,2 metros del nivel del mar, destacando las marismas naturales y las transformadas en salinas.

Este efecto incrementa la erosión costera en las playas de la Bahía de Cádiz. Un caso destacable es el de la flecha de Valdelagrana, en la zona sur de la playa de Levante (El Puerto de Santa María), donde la línea costera ha ido retrocediendo $(6,2 \mathrm{~m} /$ año $)$ ocasionando la pérdida de gran parte de playa, dunas y marismas que conforman el Parque Natural (Benavente et al., 2015). Por otro lado, las marismas y salinas

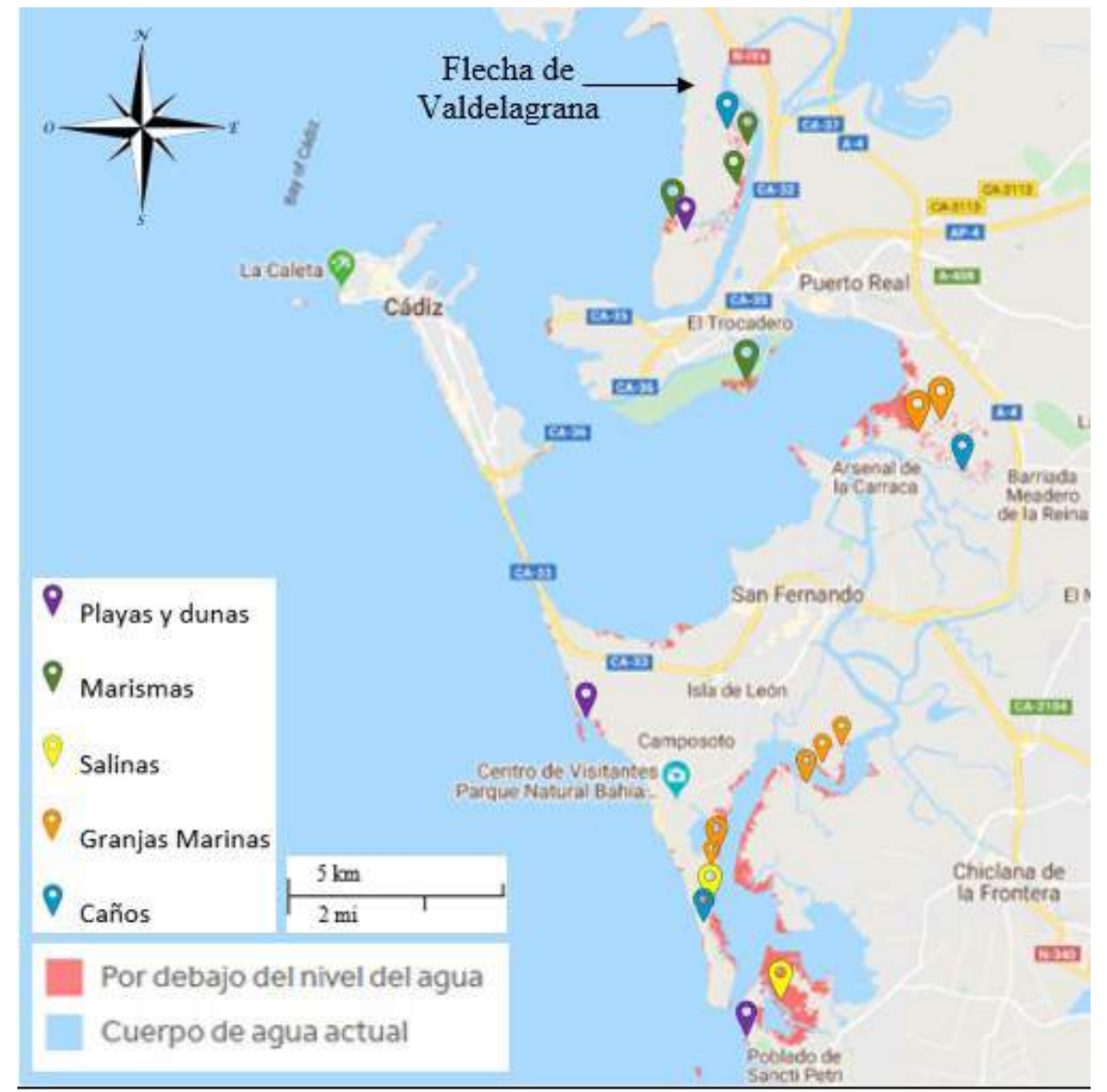

Figura 4. Mapa satélite de la Bahía de Cádiz en una simulación del terreno por debajo de 0,2 metros. Fuente: A partir de Climate Central ( 2020).

Figure 4. Satellite maps of the Bay of Cadiz in the land simulation below 0,2 meters. Source: Own elaboration by Climate Central (2020). 


\section{rin \\ COSTAS}

son elementos vitales en la biodiversidad de la bahía. Los ecosistemas son esenciales para la nidificación y migración de aves o el abastecimiento de alimentos. Además, en cuanto a los fondos marinos, las praderas de fanerógamas pueden verse afectadas por esta subida ya que algunas especies se localizan en aguas poco profundas. Si se produce una subida del nivel del mar en cortos periodos de tiempo puede verse perjudicada la capacidad de adaptación a estos cambios.
Por otra parte, con respecto al parámetro tiempo, se ha observado como gran parte de los ecosistemas del Parque Natural quedarían totalmente inundados. Podría decirse que, en 2050, buena parte de la Bahía de Cádiz estaría inundada según se muestra en la figura 5.

No obstante, teniendo en cuenta que son datos provisionales y que forman parte de una simulación a través de los datos obtenidos de años anteriores, se

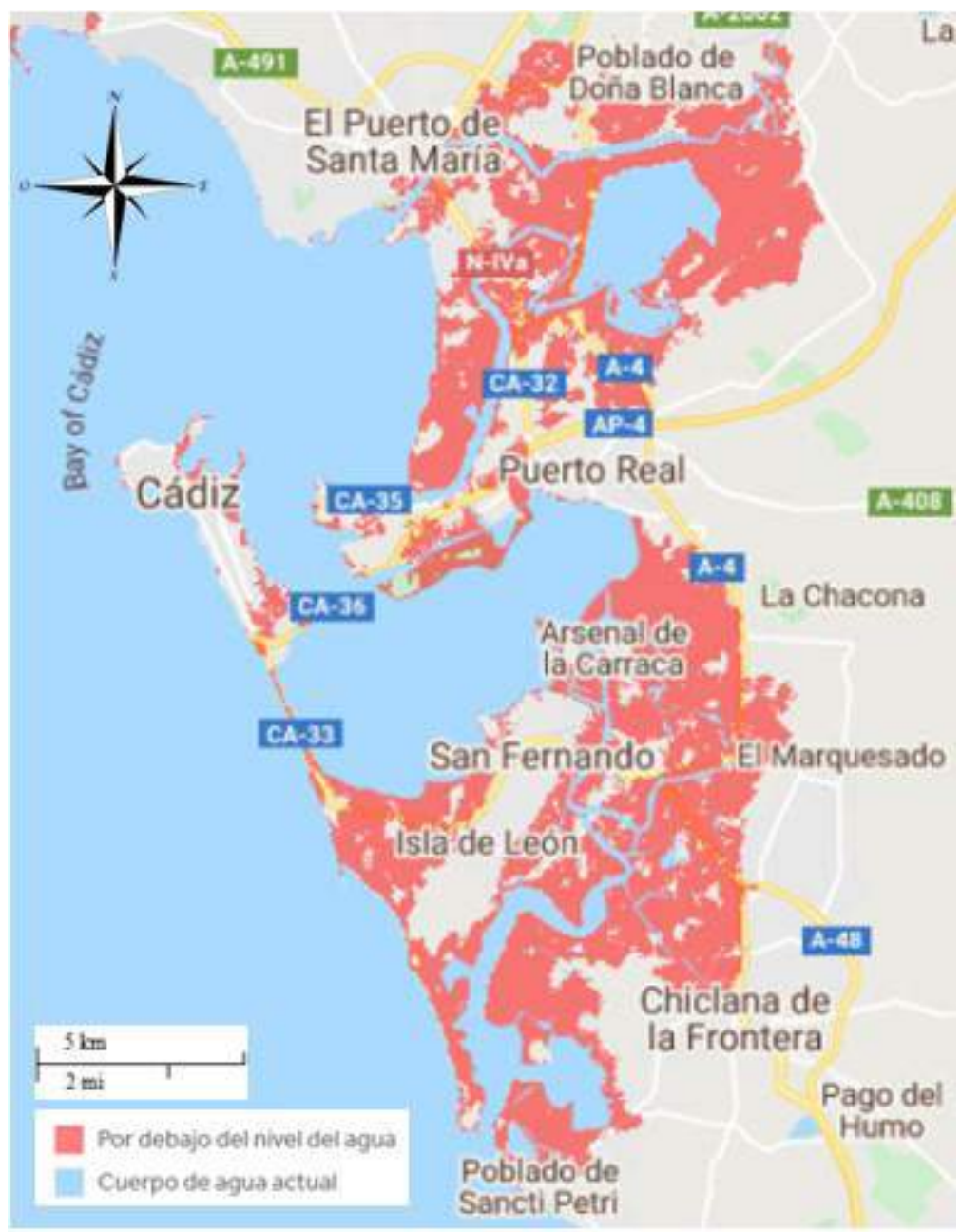

Figura 5. Mapa satélite de la Bahía de Cádiz en una simulación del año 2050. En esta figura se muestra cómo cambiaría el nivel del mar en ese año, quedando zonas terrestres totalmente inundadas. Fuente: A partir de Climate Central (2020).

Figure 5. Satellite maps of the Bay of Cadiz in a simulation date from 2050. In this figure shows how the sea level would change in this year, resulting completely flooded land areas. Source: Own elaboration by Climate Central (2020). 
infiere que, si se compara con el mapa de los servicios ecosistémicos que ofrece la zona (figura 2), en un rango de 30 ańos gran parte se verían influenciados, quedando por debajo del nivel del mar. Las áreas más

\section{Conclusiones}

El uso del Parque Natural Bahía de Cádiz y su área de influencia es fundamentalmente recreativo, sobre todo turismo de sol y playa y actividades deportivas acuáticas.

Los servicios ecosistémicos de abastecimiento tienen una relación directa con la economía del entorno. Se basan principalmente en la explotación pesquera, la acuicultura y el marisqueo de la zona. Los de regulación, son esenciales para el buen funcionamiento de los ecosistemas y para el suministro de los servicios de abastecimiento y culturales. El servicio hídrico es uno de los más degradados junto al control biológico. Sin embargo, los servicios culturales se encuentran en buen estado, destacando la educación ambiental y el desarrollo científico, disfrute paisajístico o el turismo y recreación. En general, los servicios que presentan una tendencia negativa son los de regulación, al igual que los de abastecimiento tradicionales. No obstante, los de abastecimiento industriales como la acuicultura, y los culturales están aumentando.

\section{Referencias}

Alonso, C., Gracia, F. J. \& Benavente, J. (2009). Evolución histórica de la línea de costa en el sector meridional de la Bahía de Cádiz. Revista Atlántica-Mediterránea de Prehistoria y Arqueología Social, 11: 13-37.

Anderson, E.P., Marengo, J.A., Villalba, R., Halloy, S.R.P., Young, B.E., Cordero, D., Gast, F., Jaimes, E. \& Ruíz, D. (2012). Consecuencias del Cambio Climático en los Ecosistemas y Servicios Ecosistémicos de los Andes afectadas serán las marismas y salinas de San Fernando junto a la zona del poblado de Sancti Petri en Chiclana, las marismas en la playa de Levante y algunas franjas de El Puerto de Santa María.

Los servicios que se obtienen de las fanerógamas marinas, situadas en los fondos marinos de la Bahía de Cádiz, hace replantearse que deberían estar dentro de los límites del Parque Natural.

Finalmente, se aprecian numerosas consecuencias asociadas al cambio climático. La zona costera del Parque Natural es sensible a la subida de las temperaturas y del nivel del mar, ocasionando inundaciones o retrocesos costeros que generarán pérdidas en los servicios ofrecidos por los ecosistemas. Con una visión de futuro, existe la necesidad de elaborar medidas determinadas para los cambios que tienen lugar ahora y en la posterioridad. En Espańa y otros países de Europa, ya se aplican medidas mitigadoras como el control de emisiones, promoción de energías renovables, etc. para reducir estas consecuencias, pero surge la necesidad de establecer estrategias adaptativas a escala municipal y subregional como es su consideración en la planificación urbanística y en la ordenación del territorio, para los acontecimientos que ya se están dando.

Tropicales. Herzog, S.K., R. Martinez, P.M. Jorgensen y H. Tiessen (eds.). Cambio Climático y Biodiversidad en los Andes Tropicales, Parte I, Capítulo 1: 1-22.

Barragán, J.M. \& de Andrés, M. (2020). The management of the socio-ecological systems of the Bay of Cádiz: new public policies with old instruments? Boletin de la Asociación de Geógrafos Españoles, 85: 1-42. 


\section{th \\ COSTAS}

Benavente, J., del Río, L. \& Gracia, F.J. (2015). Riesgos de erosión costera en el litoral de Cádiz: Problemática actual y perspectivas futuras. En J. \&. Rodríguez, El litoral de Andalucía. Norma y naturaleza (65-91). Universidad de Huelva.

Caballero, M., Lozano, S. \& Ortega, B. (2007). Efecto invernadero, calentamiento global y cambio climático: una perspectiva desde las ciencias de la tierra. Revista Digital Universitaria, Vol. 8, 10:1-11.

Ceballos, A., Morán, E. \& Quirós, M. (2007). Evolución de las temperaturas y precipitaciones en las capitales de Castilla y León en el periodo 1961-2006. POLÍGONOS. Revista de Geografía, 17: 59-81.

Chica, J. A., Barragán, J. M. \& Borja, F. (2011). Estado y tendencia de los servicios de los ecosistemas litorales de Andalucía. Universidad de Cádiz, 112 pp.

CAGPDS. (2019). $1^{\circ}$ Borrador del II Plan de Desarrollo Sostenible del Parque Natural Bahia de Cádiz y de su Área de Influencia Socioeconómica. Junta de Andalucía, $235 \mathrm{pp}$.

Costanza, R., de Groot, R., Braat, L., Kubiszewski, I., Fioramonti, L., Sutton, P., Farber, S. \& Grasso, M. (2017). Twenty years of ecosystem services: How far have we come and how far do we still need to go? Ecosystem Services, 28: 1-26.

Costanza, R., de Groot, R., Sutton, P., van der Ploeg, S., Anderson, S. J., Kubiszewski, I., Farber, S. \& Turner, R. K. (2014). Changes in the global value of ecosystem services. Global Envir. Change, 26: 152-158.

de Andrés, M., Barragán, J.M. \& García, J. (2018). Ecosystem services and urban development in coastal Social-Ecological Systems: The Bay of Cádiz case study. Ocean and Coastal Management, 154: 155-167.

Díaz, G. (2012). El Cambio Climático (Climate Change). Ciencia y Sociedad, Volumen XXXVII, 2: 227-240.

García-López, S., Ruiz-Ortiz, V., Barbero, L. \& Sánchez-Bellón, A. (2018). Contribution of the UAS to the determination of the water budget in a coastal wetland: a case study in the natural park of the Bay of Cádiz (SW Spain). European Journal of Remote Sensing, 51:1, 965-977.

Gómez García, J. C. (2011). Figuras de protección de la naturaleza $1,18 \mathrm{pp}$.

González, M., Jurado, E., González, S., Aguirre, O., Jiménez, J. \& Navar, J. (2003). Cambio Climático Mundial: Origen y consecuencias. CIENCIA UANL. VOL. VI, 3: 377-386.
Revista Costas, Vol. 2 (2), 2020

Haines-Young, R. \& Potschin, M. (2013). Common International Classification of Ecosystem Services (CICES) V5.1 Guidance on the Application of the Revised Structure. Nottingham, UK: Fabis Consulting Ltd., 34 pp.

Haines-Young, R. \& Potschin, M. (2018). Common International Classification of Ecosystem Services (CICES) V5.1 Guidance on the Application of the Revised Structure. Nottingham, UK: Fabis Consulting Ltd., 53 pp.

Hernández, I., Morris, E., Vergara, J.J., de los Santos, C.B., González-Ortiz, V., Villazán, B., Peralta, G., Olivé, I., Brun, F.G., García-Marín, P., Lara, M. \& Pérez-Lloréns, J.L. (2010). Praderas de fanerógamas marinas en la bahía de Cádiz: conservación y gestión. Conama10. Congreso Nacional del Medio Ambiente, $13 \mathrm{pp}$.

López, J. (2001). El aprovechamiento de los recursos naturales en el Parque Natural Bahía de Cádiz. PH, Boletín 35: 167-171.

Martín-López, B., Gómez-Baggethun, E. \& Montes, C. (2009). Un marco conceptual para la gestión de las interacciones naturaleza - sociedad en un mundo cambiante. CUIDES (Cuaderno Interdisciplinar de Desarrollo Sostenible), 3: 229-258.

Millennium Ecosystem Assessment. (2005). Ecosystems and Human Well-Being: Synthesis. Washington, DC: Island Press, 55 pp.

PNACC. (2020). Plan Nacional de Adaptación al Cambio Climático 2021-2030 Borrador 30 abril 2020. Ministerio para la Transición Ecológica y el Reto Demográfico, Gobierno de España, 79 pp.

PORN. (2004). ANEXO I Plan de Ordenación de los Recursos Naturales. Decreto 79/2004, de 24 de febrero, por el que se aprueban el Plan de Ordenación de los Recursos Naturales y el Plan Rector de Uso y Gestión del Parque Natural Bahía de Cádiz, 102 pp.

Reyes, J.L., Benavente, J., Gracia, F.J. \& López-Aguayo, F. (1996). Efectos de los temporales sobre las playas de la Babia de Cádiz. IV Reunión de Geomorfología, Grandal d'Anglade, A. y Pagés Valcarlos, J., Eds. O Castro, A Coruña: Sociedad Española de Geomorfología, 631-643.

Rodríguez, J., Reul, A., Blanco, J.M. \& Rodríguez, V. (2011). Estado y tendencia de los servicios de los ecosistemas marinos de aguas exteriores de Andalucia. Universidad de Málaga, 74 pp.

TEEB. (2010). The Economics of Ecosystems and Biodiversity. Mainstreaming the economics of nature. A synthesis 
of the approach, conclusions and recommendations of TEEB. Malta: Progress Press, 39 pp.

Tolón Becerra, A. \& Lastra Bravo, X. (2008). Los espacios naturales protegidos. Concepto, evolución y situación actual en España. RevistaElectrónic@de Medioambiente, 5: 1-25.

UNEP. (2009). Ecosystem Management Programme. A new approach to sustainability. Nairobi, Kenya: United $\mathrm{Na}-$ tions Environment Programme, 24 pp.

\section{Otras fuentes de información}

AEMET. (2020). Resúmenes climatológicos. Andalucía. 2020, de AEMET: Agencia Estatal de Meteorología Sitio web:

http://www.aemet.es/es/serviciosclimaticos/vigilancia_cli$\mathrm{ma} /$ resumenes? $\mathrm{w}=1 \&$ datos $=-1 \& \mathrm{n}=1 \& \mathrm{k}=$ and

AEMET. (2020). Valores climatológicos normales. Cádiz. 2020, de AEMET: Agencia Estatal de Meteorología Sitio web:

http://www.aemet.es/es/serviciosclimaticos/datosclimatologicos/valoresclimatologicos?l=5973\&k=undefined

Climate Central. (2020). CoastalDEM. Coastal Risk Screening Tool. 2020, de Climate Central: A Science $\&$ News Organization. Sitio web:

ht tps:// coastal.climatecentral.org/ map/11/6.0907/36.4822/?theme=sea_level_rise\&map_type=year\&contiguous=true\&elevation_model=best_available\&forecast_year $=2100 \&$ path way=rcp 45\&percentile=p50\&return_level=return_ level_1\&slr_model=kopp_2014

PSMSL. (2019). Obtención de datos del indicador de mareas. Mareógrafo Cádiz III. 2020, de PSMSL: Permanent Service for Mean Sea Level. Sitio web: https://www.psmsl.org/data/obtaining/stations/985.php 


\section{cos \\ COSTAS}

Revista Costas, Vol. 2 (2), 2020

$>2004$ 